\title{
Hadronization, chemical equilibrium and chemical freeze-out
}

\section{F. Becattini*}

University of Florence and INFN

Department of Physics and Astronomy, Via G. Sansone 1, I-50019, Sesto F.no (Firenze), Italy

E-mail: becattini@fi.infn.it

\section{Eduardo Grossi}

Università di Firenze and INFN Sezione di Firenze, Firenze, Italy

\section{Jan Steinheimer}

Frankfurt Institute for Advanced Studies (FIAS), Frankfurt, Germany

\section{Reinhard Stock}

Institut für Kernphysik, Goethe-Universität, Frankfurt, Germany

\section{Marcus Bleicher}

Frankfurt Institute for Advanced Studies (FIAS), Frankfurt, Germany

I review recent results in the subject of hadron production in relativistic heavy ion collisions and the concepts of chemical freeze-out, chemical equilibration and the role of post-hadronization inelastic collisions at the LHC energy.

9th International Workshop on Critical Point and Onset of Deconfinement - CPOD2014,

17-21 November 2014

ZiF (Center of Interdisciplinary Research), University of Bielefeld, Germany

\footnotetext{
${ }^{*}$ Speaker.
} 


\section{Introduction}

The study of hadroproduction in relativistic heavy collisions has been the subject of an intense research work for more than 20 years by now. Accurate measurements of multiplicities of the different species and their spectra over a large span of centre-of-mass energies have been a major test bench for the models and have led to a concrete verification of the QCD phase diagram. Recently, the accurate measurements provided by the LHC experiments at a nucleon-nucleon centre of mass energy of $\sqrt{s}_{N N}=2.75 \mathrm{TeV}$ have renewed the interest in the dynamics of the bulk hadron production.

The collected evidence in elementary and heavy ion collisions points to the following picture of the process of hadron production:

- Hadrons are produced at local equilibrium at a pseudo-critical temperature because of some intrinsic feature of non-perturbative QCD, possibly related to the so-called quantum thermalization phenomenon [1];

- Hadron reinteraction is possible if the hadronizing system is large enough, like in heavy ion collisions. Hadronic collisions in the expanding system can drive it out of equilibrium until freeze-out occurs;

It is possible to model post-hadronization dynamics (afterburning) with some numerical code such as UrQMD, hence to reconstruct the primordial equilibrium conditions of the hadronic system, what we define as LCEP, Latest Chemical Equilibrium Point. Although conceptually different from the hadronization point (hadrons can reinteract for a very short time cooling down while maintaining chemical equilibrium), it seems reasonable to assume LCEP virtually coinciding with hadronization.

\section{The freeze-out process}

In an expanding sytem of interacting particles freeze-out occurs when the mean scattering time $\tau_{\text {scatt }}$ exceeds the mean collision time $\tau_{\text {exp }}$ :

$$
\tau_{\text {scatt }}=\frac{1}{n \sigma\langle v\rangle}>\tau_{\exp }=\frac{1}{\partial \cdot u}
$$

$u$ being the hydrodynamical velocity field and $\langle v\rangle$ is the mean velocity of particles. If the crosssection $\sigma$ is the inelastic one, the freeze-out is called chemical, whereas if it includes elastic processes, the freeze-out is called kinetic. Chemical freeze-out of course precedes the kinetic as the inelastic cross section is smaller than the total.

We can obtain a gross approximation of the expansion time with the ratio $V / \dot{V}$ where $V(t)$ is the volume of the fireball at the time $t$. For a fireball which is spherical in shape with a radius $R$, this is $R / 3 \dot{R}$ and if the radius increases at approximately the mean particle velocity $\langle v\rangle$, we have the condition:

$$
\frac{1}{n \sigma\langle v\rangle}>\frac{R}{3\langle v\rangle} \Longrightarrow \frac{1}{n \sigma}>\frac{R}{3}
$$


For $N$ particles within the volume, this inequality yields the radius at which freeze-out occurs as a function of $N$ and of the average cross-section:

$$
R_{\mathrm{fo}}=\sqrt{\frac{N \sigma}{4 \pi}}
$$

and the density at which freeze-out occurs, which decreases with $N$ according to:

$$
n_{\mathrm{fo}}=\frac{N}{\frac{4 \pi}{3} R_{\mathrm{fo}}^{3}}=3 \sqrt{\frac{4 \pi}{N}} \frac{1}{\sigma^{3 / 2}}
$$

Of course, it should be kept in mind these estimates (2.3) and (2.4) are crude, but they tell us that the freeze-out radius, for each particle, approximately scales with the square root of the number of scattering centers a particle can interact with and the related cross section. For a low multiplicity hadronic system, it may happen that the above value exceeds the density of hadrons when they are formed, that is at hadronization. This simply signals that hadrons decouple right after their formation without reinteracting, what happens in elementary collisions, at the intrinsic hadronization density scale which is dictated by QCD. For relativistic heavy ion collisions, conversely, the multiplicity can grow to large numbers so that there could be enough time for hadronic reinteraction and freeze-out occurs later. For instance, for the typical value of $N=1000$, in most central collisions, and $\sigma=30 \mathrm{mb}=3 \mathrm{fm}^{2}$ one has $R_{\mathrm{fo}} \simeq 15 \mathrm{fm}$, which is in the right ballpark (for kinetic freeze-out) taking into account the drastic approximations made; the density at freeze-out turns out to be $n_{\mathrm{fo}} \simeq 0.06 \mathrm{fm}^{-3}$ which is lower than the typical hadronization density of about $0.5 \mathrm{fm}^{-3}$.

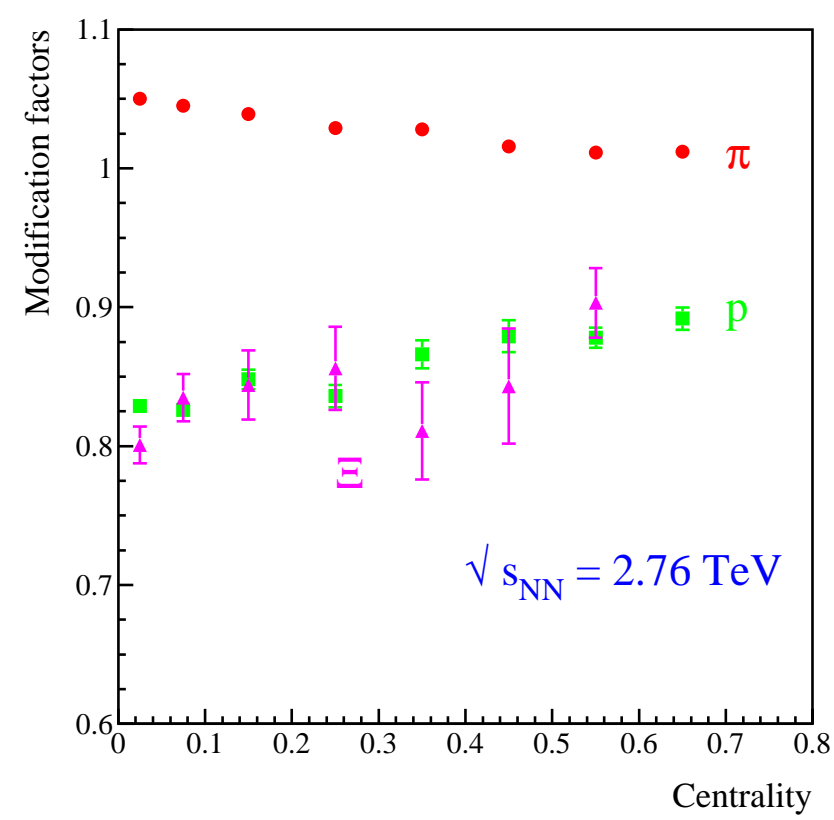

Figure 1: Modification factors (see text for definition) for $\pi^{+}$, proton, and $\Xi^{-}$as a function of centrality at $\sqrt{s N}_{\mathrm{NN}}=2.76 \mathrm{TeV}$ calculated with UrQMD. The error bars are statistical. From ref. [2]. 


\section{An analysis of yields at different centralities at the LHC}

As the freeze-out conditions depend on multiplicity, one should then expect that the amount of post-hadronization collisions depends on centrality at some fixed centre-of- mass energy. Indeed, while in central collisions multiplicity is large and there should then be, e.g., more antibaryon baryon annihilation/regeneration than in peripheral collisions. Thus, if our hypothesis is correct that the QCD hadronization process generates an equilibrium hadron/resonance yield distribution, at some constant temperature $T$, the afterburning effects should lead to a larger modification in central than in peripheral collisions. As baryon attenuation leads to lower apparent freeze-out temperatures derived from the standard SHM analysis, we would expect this temperature to rise, mildly, from central toward peripheral collisions. We have analyzed the multiplicities measured

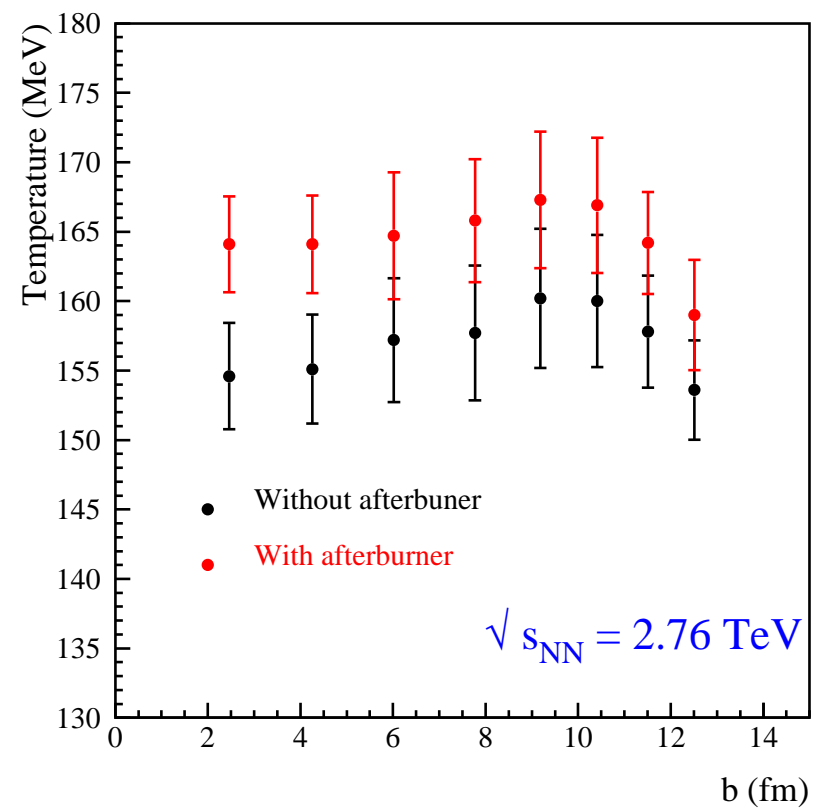

Figure 2: Temperature as a function of the impact parameter $b$ (central values corresponding to centralities measured by ALICE). Black dots: chemical freeze-out temperature. Red dots: LCEP (see text) temperature obtained by including UrQMD modification factors. From ref. [2].

by the ALICE experiment at $\sqrt{s}_{\mathrm{NN}}=2.76 \mathrm{TeV}$ [3] to determine the chemical freeze-out parameters with fits to the usual SHM predictions and to the same formulae corrected for the modification factors, defined as the ratios between the particle yields with afterburning and the same yields without it. Details of the analysis can be found in ref. [2]. The modification factors (see fig. 1 have been estimated with a hybrid version of the code UrQMD [4] implementing afterburning after a hadron generation according to local thermodynamical equilibrium prescription (Cooper-Frye formula).

The results of the fit are shown in fig. 2 and 3. As can be seen from fig. 3, the fit quality improves throughout after the implementation of afterburning corrections. The fitted temperature 


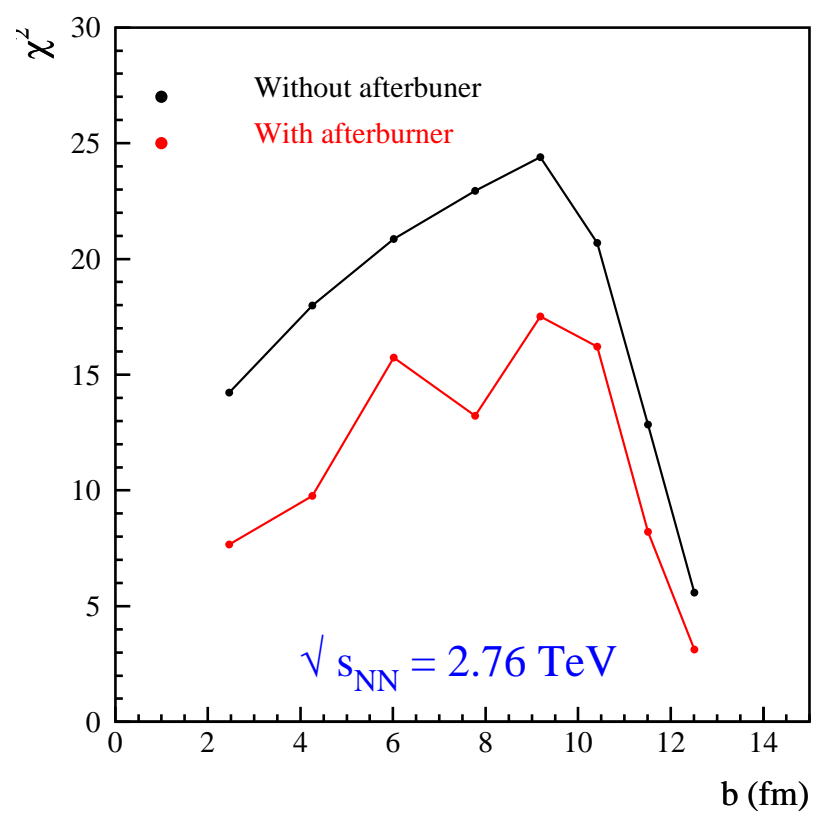

Figure 3: (Color online) $\chi^{2}$ of the SHM fits with and without afterburning corrections as a function of the impact parameter $b$ (central values corresponding to centralities measured by ALICE). The fitted parameters being in this case $T, \gamma_{S}$ and the normalization, the number of degrees of freedom is 7. From ref. [2].

rises by several MeV's, as shown in fig. 2, in agreement with previous findings [5]. Furthermore, the LCEP temperature is less centrality dependent than the plain chemical freeze-out temperature, which bears out the idea of a universal (at fixed baryon density) hadronization temperature [6, 7]. This is best seen in fig. 4 where we show the difference between the corrected temperature and the plain SHM fitted one. The difference steadily decreases towards peripheral collisions, again in full agreement with the picture that afterburning affects less the chemical composition if the overall multiplicity is lower. There remain two small structures in the temperature vs centrality plot after the afterburning correction: a mild rise towards mid-peripheral collisions (see fig. 2) and a sizeable decrease in most peripheral collisions.

\section{Conclusions}

To summarize, we have demonstrated that in the high multiplicity environment of relativistic heavy ion collisions at $\sqrt{s}_{\mathrm{NN}}=2.76 \mathrm{TeV}$ the inelastic collisions play a significant role in modifying the primordial hadronic yields from hadronization. The amount of inelastic rescattering is expected to depend on multiplicity, hence on centrality. This effect is clearly seen in the centrality dependence of specific particle ratios measured by the ALICE experiment and especially $\Xi / \pi$ which - for the first time - is observed to increase towards peripheral collisions before dropping. These findings are in excellent agreement with the concept of a universal statistical hadronization occurring at the pseudo-critical QCD temperature. 


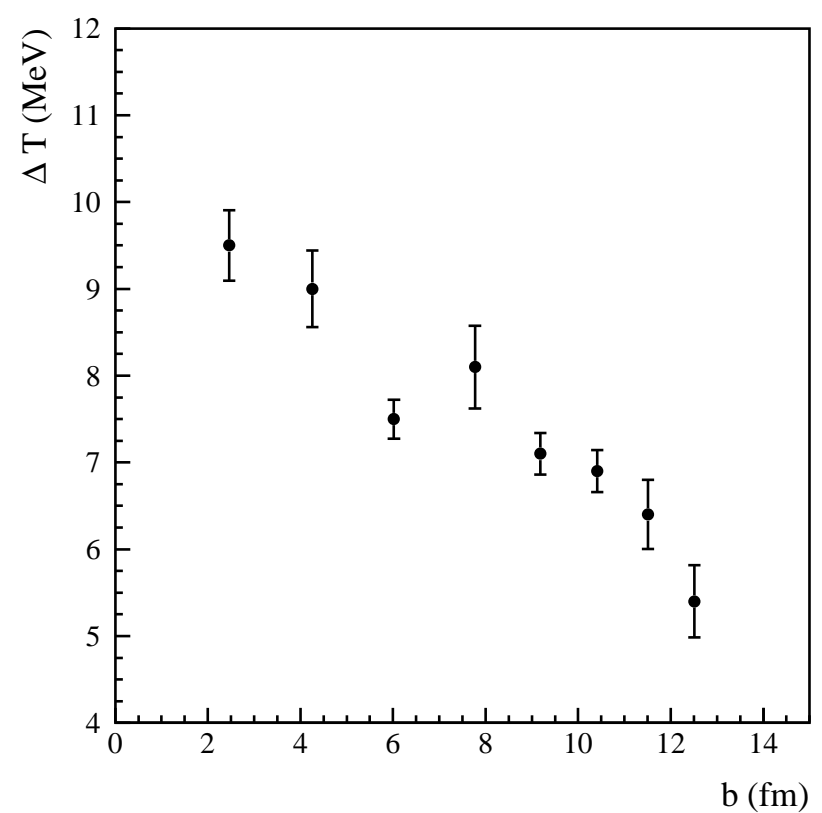

Figure 4: Difference between the corrected temperature and the chemical freeze-out temperature as a function of the impact parameter $b$ (central values corresponding to centralities measured by ALICE). The error bar has been estimated by taking a $100 \%$ correlation between the errors on $T$ in the two fits. From ref. [2].

\section{Acknowledgments}

F. Becattini would like to thank the organizers for their kind hospitality and excellent work in arranging the CPOD conference.

\section{References}

[1] M. Srednicki, Phys. Rev. E 50 (1994) 888.

[2] F. Becattini, E. Grossi, M. Bleicher, J. Steinheimer and R. Stock, Phys. Rev. C 90 (2014) 5, 054907.

[3] B. B. Abelev et al. [ALICE Collaboration], Phys. Lett. B 728,(2014) 216; B. B. Abelev et al. [ALICE Collaboration], Phys. Rev. Lett. 111, (2013) 222301 ; B. Abelev et al. [ALICE Collaboration], Phys. Rev. C 88, (2013) 044910.

[4] S. A. Bass et al., Prog. Part. Nucl. Phys. 41, 255 (1998);

M. Bleicher et al., J. Phys. G25, 1859 (1999);

H. Petersen, J. Steinheimer, G. Burau, M. Bleicher and H. Stöcker, Phys. Rev. C 78, 044901 (2008);

H. Petersen, M. Bleicher, S. A. Bass and H. Stocker, arXiv:0805.0567 [hep-ph].

[5] F. Becattini, M. Bleicher, T. Kollegger, T. Schuster, J. Steinheimer and R. Stock, Phys. Rev. Lett. 111, 082302 (2013).

[6] F. Becattini, arXiv:0901.3643 [hep-ph] and references therein.

[7] H. Satz, Int. J. Mod. Phys. E 21, 1230006 (2012) and references therein. 